\title{
Phase and glass transitions in short-range central potential model systems: the case of $\mathrm{C}_{60}$
}

\author{
Maria C. Abramo, Carlo Caccamo*, Dino Costa, and Romina Ruberto \\ Dipartimento di Fisica, Università degli Studi di Messina \\ Contrada Papardo, C.P. 50 - 98166 Messina, Italy
}

\begin{abstract}
Extensive molecular dynamics simulations show that a short-range central potential, suited to model $\mathrm{C}_{60}$, undergoes a high temperature transition to a glassy phase characterized by the positional disorder of the constituent particles. Crystallization, melting and sublimation, which also take place during the simulation runs, are illustrated in detail. It turns out that vitrification and the mentioned phase transitions occur when the packing fraction of the system — defined in terms of an effective hard-core diameter - equals that of hard spheres at their own glass and melting transition, respectively. A close analogy also emerges between our findings and recent mode coupling theory calculations of structural arrest lines in a similar model of protein solutions. We argue that the conclusions of the present study might hold for a wide class of potentials currently employed to mimic interactions in complex fluids (some of which of biological interest), suggesting how to achieve at least qualitative predictions of vitrification and crystallization in those systems.
\end{abstract}

\section{INTRODUCTION}

Central, short-range potential models of simple analytic form have been the object of intense investigation over the last years. The main reason of such an interest is that they provide an approximate representation of the effective interactions and phase behavior experimentally observed in a variety of macrosized particle systems, spanning from colloidal suspensions and protein or polymer solutions $1,2,43,4,5,6,7,8$ to other macromolecular systems and fullerenes $[9,10,11,12,13,14]$. A paradigmatic example in this respect is offered by protein crystallization [15, 16, 17]: indeed, if one adopts a representation in which the macromolecules are spherical particles interacting through a central short-range potential, and the solvent is assumed to be a structureless continuum, the liquid-vapor binodal and the sublimation line thereby calculated exhibit all features of the proteinrich/protein-poor and crystallization line observed in real globular protein solutions. These models then offers an unvaluable tool for the study, by usual means of statistical mechanics and simulation, of the relative location of phase coexistence lines in real systems.

This scenario has been further enriched by recent investigations of glass transition in one of the most studied short-range model, namely the attractive hard-core Yukawa fluid. In this context the mode coupling theory predicts the onset of two glassy phases deeply inside the metastable liquid-solid region of the model [7]. The formation of distinct vitreous phases with peculiar internal characteristics has also been reported in recent experiments on colloid-polymer mixtures 18, 19, 20].

These evidences prompted us to an extensive investigation of phase coexistence and glass transition conditions in short-range models, with particular attention to the

${ }^{*}$ Email: carlo.caccamo@unime.it rich phenomenology which characterizes the metastable region enveloped between the freezing and the melting line of such systems. The paper focuses in particular on the Girifalco potential for fullerene $\mathrm{C}_{60}$ 21]. This model can be considered as "marginally" short-range in the sense that, whereas in colloidal and protein systems the interaction between (model) macroparticles reduces practically to zero within a fraction of the particle diameter and the liquid-vapor equilibrium is only metastable, in the Girifalco model the decay length of interactions is slightly greater than the fullerene diameter and a stable liquid phase does exist, albeit confined to a temperature interval smaller than $100 \mathrm{~K}$ [9, 12, 13, 14], with no experimental evidence of it hitherto reported [22, 23, 24, 25, 26]. Such characteristics appear consistent with early speculations about the "elusive diffusive" nature of liquid $\mathrm{C}_{60}$ 11], as well as with recent evidences of the absence, in this peculiar fluid, of cage effects in the velocity autocorrelation functions, and of collective phenomena, ordinarily observed in simple liquids 27].

We have recently reported in a preliminary communication [28] (hereafter referred to as I) Molecular Dynamics (MD) evidences of the onset of a glass transition in $\mathrm{C}_{60}$ at $T=1100 \mathrm{~K}$ upon a pressure of $3.5 \mathrm{MPa}$, as associated to positional disorder. Such a glassy phase is quite distinct from the experimentally detected $\mathrm{C}_{60}$ orientational glass, which forms at $90 \mathrm{~K}$ as a consequence of the freezing at low temperature of the residual disorder in the orientation of the fullerene cages [29, 30].

As preliminary documented in I, vitrification of $\mathrm{C}_{60}$ at high temperature is assessed through quenching cycles of the liquid phase at various pressures, by analyzing a number of thermodynamic, structural and dynamical quantities. Here, we report and discuss in detail the MD results we cumulated to support our conclusion that a positional glass of $\mathrm{C}_{60}$ is effectively formed. In the same context, we discuss other transitions undergone by the system in the course of cooling or heating cycles, such as crystallization, melting and sublimation. As we shall detail, 
such transitions take place deeply inside the metastable region in coincidence of the crossing of two density-vstemperature loci over which the "effective" packing fraction of the system equals that of hard spheres at their melting and glass transition, respectively. The analysis of such hard-sphere-like behavior enlights the analogies of our study with the case, discussed in reference [7], of the onset of glassy phases in model protein solutions, suggesting that our results can be useful in the more general context of complex fluids investigation.

The paper is structured as follows: in section II we introduce the model and describe the computational strategies. In III results for thermodynamics, structural and dynamical properties are reported. The discussion and conclusions follow in section IV.

\section{MODEL AND SIMULATION STRATEGIES}

We study a system composed of particles interacting via the Girifalco potential 21],

$$
\begin{aligned}
v(r)=- & \alpha_{1}\left[\frac{1}{s(s-1)^{3}}+\frac{1}{s(s+1)^{3}}-\frac{2}{s^{4}}\right] \\
+ & \alpha_{2}\left[\frac{1}{s(s-1)^{9}}+\frac{1}{s(s+1)^{9}}-\frac{2}{s^{10}}\right]
\end{aligned}
$$

where $s=r / d, \alpha_{1}=N^{2} A / 12 d^{6}$, and $\alpha_{2}=N^{2} B / 90 d^{12}$; $N=60$ and $d=0.71 \mathrm{~nm}$ are the number of carbon atoms and the diameter, respectively, of the fullerene particles, $A=32 \times 10^{-60} \mathrm{erg} \mathrm{cm}^{6}$ and $B=55.77 \times 10^{-105} \mathrm{erg} \mathrm{cm}^{12}$ are constants entering the 12-6 potential $\phi(\mathrm{r})=-A / r^{6}+$ $B / r^{12}$ through which two carbon sites on different spherical molecules are assumed to interact. The distance where the potential (11) crosses zero, the position of the potential well minimum and its depth, are $\sigma=0.959 \mathrm{~nm}$, $r_{\min }=1.005 \mathrm{~nm}$, and $\varepsilon=0.444 \times 10^{-12} \mathrm{erg}$, respectively [21]. We assume $v(r)=\infty$ for $s<1$.

We have used the MD method introduced by Andersen 31] to simulate constant-pressure, constant-enthalpy $(\mathrm{NPH})$ conditions, and the Verlet algorithm to integrate the equations of motion over time steps $\Delta t=5 \mathrm{fs}$; cubic boxes and periodic boundary conditions are assumed. Most results are obtained for $N=864$ and $N=1000$ total number of particles; the smaller sample is fully compatible with an initial fcc lattice arrangement of the particles in a cubic simulation box, whereas the 1000-particle distribution obviously implies defects. Both sizes are part of a more general analysis employing also five hundred and 1372 particles, to exclude any dependence of simulation results on the sample size. A wide range of pressures, spanning from close-to-atmospheric conditions up to $250 \mathrm{MPa}(2.5 \mathrm{kbar})$, has been explored to verify the effects on crystallization and glass transition conditions. Cooling and quenching cycles start from an initial supercritical or liquid configuration (see figure 1), identified as such on the basis of the known phase diagram of $\mathrm{C}_{60}$ [14]. Heating cycles begin either from the defective crystals eventually obtained at the end of the cooling cycles, or from a perfect fcc crystal at room temperature with 864 particles. Other details are given in the presentation of results.

\section{RESULTS}

For clarity sake, we shall detail in the first instance the transitions undergone by the system during slow cooling or heating sequences, followed by a presentation of results obtained during the quenching routines. Just before, we introduce the concept of "effective" packing fraction for the system at issue, with two associated loci of thermodynamic states.

\section{A. Effective hard sphere diameters and constant "packing" loci}

We introduce an effective hard sphere diameter for the $\mathrm{C}_{60}-\mathrm{C}_{60}$ interaction with the definition of a reference potential for $v(r)$ of equation (11) according to the well-known Weeks-Chandler-Andersen (WCA) prescription [32],

$$
v_{\mathrm{ref}}(r)=\left\{\begin{array}{cc}
v(r)+\varepsilon & \text { if } r \leq r_{\min } \\
0 & \text { if } r>r_{\min }
\end{array}\right.
$$

and adopt the Barker and Henderson expression for the effective hard-core diameter [33],

$$
\sigma_{\mathrm{BH}}=\int_{0}^{\infty}\left\{1-\exp \left[-\beta v_{\mathrm{ref}}(r)\right]\right\} d r .
$$

The Barker-Henderson prescription for the hard sphere diameter is by no means unique. Other more sophisticated recipes, hinging for instance on the use of the cavity distribution function $y(r)=\exp [\beta v(r)] g(r)$ 34] (where $g(r)$ is the radial distribution function), have been proposed in the literature. We have verified however that the resulting changes in the estimate of the effective diameter with respect to the simpler $\mathrm{BH}$ prescription are quite minor.

We now look for densities $\rho=\rho(T)$ which make the effective packing fraction $\eta=\pi / 6 \rho \sigma_{\mathrm{BH}}^{3}$ constant with respect to temperature variations, and equal to a prefixed value. In particular, we impose $\eta$ to be equal to the packing of hard spheres at their own melting and glass transition, respectively. The value $\eta_{\mathrm{m}}^{\mathrm{HS}}=0.545$ has been reported for the packing fraction of hard spheres at melting [35]. A packing of 0.55 has also been reported in constant pressure MD simulation of hard spheres [36]. Here we choose an intermediate value (see I), by imposing

$$
\eta_{\mathrm{m}}=\pi / 6 \rho_{\mathrm{m}}(T) \sigma_{\mathrm{BH}}^{3}=0.548
$$


As for the packing of hard spheres at the glass transition, $\eta_{\mathrm{g}}^{\mathrm{HS}}$, the value 0.58 has been reported in reference [37]. whereas effective packing fractions at vitrification for continous potentials, mostly of Lennard-Joneslike form (see, e.g. 38] and references), approach 0.58 with a considerable dispersion of values due to the different prescriptions either for the effective diameter or for the determination of the glass transition temperature. We require in particular (see I):

$$
\eta_{\mathrm{g}}=\pi / 6 \rho_{\mathrm{g}}(T) \sigma_{\mathrm{BH}}^{3}=0.574 .
$$

On the basis of equations (4) and (5) we can determine the two functions $\rho_{\mathrm{m}}(T)$ and $\rho_{\mathrm{g}}(T)$ which are shown in figure 1] As we shall see, these two $\rho(T)$ loci correlate significantly with the crystallization and vitrification conditions of the $\mathrm{C}_{60}$ model.

\section{B. Cooling cycles and crystallization; heating cycles and sublimation or melting}

We first illustrate the case when the pressure $P=$ $3.5 \mathrm{MPa}$. Cooling starts from a high temperature fluid configuration at $T=2000 \mathrm{~K}$ (see figure 1 top). In sequential stepwise drops $\Delta T=30 \mathrm{~K}$, the system evolves over 20000 steps (correspondiong to 100 ps) at fixed temperature, followed by 10000 cumulation steps where the system evolves freely. The final temperature $T$, box volume $V$, enthalpy $H$, and other thermodynamic, structural and dynamical properties are recorded at each $\Delta T$ (see below). Cooling is always arrested when room temperature is achieved or sligthly before this threshold. Statistical uncertainties on $T, \rho$, and $P$ turn out to be $0.8 \mathrm{~K}$, $0.0003 \mathrm{~nm}^{-3}$ and $0.1 \mathrm{MPa}$, respectively.

Cooling paths are visualized in figure 1(top) in the $\rho-T$ representation of the phase diagram of $\mathrm{C}_{60}$. Volumes and enthalpies changes with the temperature are displayed in figure 2 (top); as visible (and already shown in I for the case $N=1000$ ), for both system sizes investigated $V$ and $H$ undergo a marked drop at $T \simeq 1300 \mathrm{~K}$, accompanied by a temporary increase of the temperature. Such a highly nonmonotonic behaviour of the two thermodynamic quantities is fully consistent with a transition of the system to the solid phase as further documented by (i) the behavior of the radial distribution function, $g(r)$ for $T \leq 1307 \mathrm{~K}$ (figure 3 top), (ii) the temperature dependence of the diffusion coefficient $D$ (figure 4 top) and (iii) the snapshots of the $\mathrm{C}_{60}$ configurations at different temperatures (figure 5). The incipient solid phase is characterized by a fcc crystalline arrangement, as visible from an inspection of the peaks' positions in the $g(r)$.

The variation of $N$ from 1000 to 864 does not produce any significant effect either on the overall cooling path, or on the location of the turning points which marks the onset of crystallization. Such an outcome is obtained if we evolve the smaller system for a longer time. In fact, under equal time elapsed conditions the turning point with 864 particles would fall at lower temperatures. We interpret
TABLE I: Crystallization $(c)$, melting $(m)$ and glass $(g)$ transition parameters. Melting data refer to the defective crystal with $N=1000$. Pressures are given in MPa, temperatures in $\mathrm{K}$, densities in $\mathrm{nm}^{-3}$, and diameters in $\mathrm{nm}$; in the last column the packing fraction $\eta_{\mathrm{x}}=\pi / 6 \rho_{\mathrm{x}} \sigma_{\mathrm{BH}}^{3}$.

\begin{tabular}{ccccc}
$P$ & $T_{\mathrm{c}}$ & $\rho_{\mathrm{c}}$ & $\sigma_{\mathrm{BH}}$ & $\eta_{\mathrm{c}}$ \\
\hline 3.5 & 1307 & 1.124 & 0.976 & 0.547 \\
40 & 1464 & 1.130 & 0.975 & 0.548 \\
150 & 1880 & 1.138 & 0.972 & 0.547 \\
250 & 2200 & 1.150 & 0.970 & 0.550 \\
$P$ & $T_{\mathrm{m}}$ & $\rho_{\mathrm{m}}$ & $\sigma_{\mathrm{BH}}$ & $\eta_{\mathrm{m}}$ \\
\hline 3.5 & 1931 & 1.144 & 0.9716 & 0.549 \\
$P$ & $T_{\mathrm{g}}$ & $\rho_{\mathrm{g}}$ & $\sigma_{\mathrm{BH}}$ & $\eta_{\mathrm{g}}$ \\
\hline 3.5 & 1100 & 1.168 & 0.978 & 0.572 \\
40 & 1170 & 1.177 & 0.977 & 0.575 \\
150 & 1480 & 1.187 & 0.975 & 0.576 \\
250 & 1700 & 1.190 & 0.973 & 0.574
\end{tabular}

this result as a manifestation of the fact, also emerging in heating cycles, that the 1000-particle case, characterized by a larger simulation box and defects with respect to the perfect fcc arrangement, easier allows for the internal particle rearrangements which trigger the phase transitions. We also note that the first of the turning points, signalling the onset of crystallization falls practically in coincidence of the crossing between the cooling path and the $\eta_{\mathrm{m}}^{\mathrm{HS}}=0.548$ locus introduced in equation (4) (compare figure 1 and figure 21); we shall discuss this point in the next section.

No appreciable effect on the system behavior is exerted by the cooling rate since cooling cycles with $\Delta T=15 \mathrm{~K}$ lead to substantially similar results (see I). Sensitive changes are instead associated to the value of the imposed pressure: the modifications undergone by the cooling paths as $P$ varies from 3.5 to 150 and $250 \mathrm{MPa}$ are shown in figure 1 (middle panel, see also the inset) and figure 2 (compare top and middle panels); the radial distribution functions for the case $P=250 \mathrm{MPa}$ are shown in the bottom panel of figure 3 and numerical values of the crystallization parameters are reported in table 【 It appears that the increase of the pressure rises dramatically the temperature where the onset of crystallization occurs. The densities attained at crystallization, and at the end of the cooling paths, are close to each other and exhibit a trend to increase with the pressure. These densities are all smaller than those of the perfect crystal heated at the corresponding temperature, indicating that defective crystals are formed. One can realize that this is so by comparing the physical characteristics of such crystals with those typical of a perfect crystal of 864 particles. We consider to this aim crystals at room temperature as previously obtained through the cooling from high temperatures of samples with $N=1000$ and $N=864$, and a perfect crystal of 864 particles under the same temper- 
ature and pressure conditions. As visible from figure 1 and figure 2 the final densities of the samples obtained from the cooling procedure are smaller than that of the perfect 864 crystal. Such lower density values must be attributed to the presence of voids in the crystalline matrix formed during the cooling process. The comparison of radial distribution functions in figure [6] enlights the smoother structure of cooled samples with respect to the perfect crystal. At $\mathrm{P}=250 \mathrm{MPa}$, after the onset of crystallization, the cooling path runs almost overlapped with the melting curve of $\mathrm{C}_{60}$ [14] (see figure 1] middle). The high pressure is thus able to force the system to evolve out of the metastable region approximately along the true coexistence line.

The transition to the crystal takes place under strong supercooling conditions, well beyond the freezing line. indeed, when the latter is crossed, no feature in the radial distribution function signals the onset of any structural order typical of the solid phase (see figure 3). Signatures albeit faint of such a crossing can be recognized however in the $V$ and $H$ patterns which show a corresponding tiny nonmonotonicity (see top panels of figure 2 and insets), as well as in the diffusion coefficient (see figure 4 and insets). As visible, runs with different number of particles exhibit the same features, ruling out the possibility that our observations are merely a consequence of statistical noise.

A similar situation emerges when the systems approaches the metastable portion of the liquid-vapor binodal of $\mathrm{C}_{60}$, calculated in reference 39] and reported in figure [1] It appears that the cooling paths get closer to the binodal the lower the pressure becomes. At $P=3.5 \mathrm{MPa}$ a crossing can be extrapolated to take place at $T=1700 \mathrm{~K}$ and $\rho=0.95 \mathrm{~nm}^{-3}$, a state point where $V$ and $H$ patterns show another nonmonotonicity, magnified in the insets of figure 2. Remarkably, also the diffusion coefficient in figure 4 is nonmotonic in correspondence of the estimated crossing of the binodal. We have carried out very long simulation runs (up to several million time steps) in correspondence of the crossing of the freezing and binodal lines, to check whether the system is able to develop any signal of the incipient transitions. In no case, however, we could monitor transformations in the thermodynamic and diffusion coefficient behavior comparable to those heralding at the crystallization temperature $T=1307 \mathrm{~K}$, as previously described. Several similarities emerge in comparison with the simulation study that two of us have performed [40] on a modified Lennard-Jones potential used to model globular protein solutions [5]. In that case, the transition to the crystalline arrangement takes place during isochoric coolings of the system deeply beneath the freezing line, when the system reaches the metastable binodal line. In the present case, the system must be cooled even substantially below the metastable binodal before crystallization can start.

Starting from the two solid simulation samples eventually obtained through the cooling cycles with $N=1000$ and 864, and from a third one built as a perfect crystal with 864 particles, we gradually heat all these systems through successive $\Delta T=30 \mathrm{~K}$ increases, up to the temperature at which they undergo an abrupt transition to a fluid configuration. Bottom panels of figures 1 and 2 refer to this sequences; it appears that the defective sample with one thousand particles undergoes a true melting since the systems jumps to a thermodynamic point inside the liquid pocket of the phase diagram whereas the 864 particle crystal, either defective or perfect, is instead substantially overheated until it jumps directly to a vapor phase, thus exhibiting sublimation. The role of defects at intermediate temperatures emerges by the comparison of the radial distribution functions obtained from the heating of the perfect crystal at relatively high temperature with the corresponding $g(r)$ of a crystal at an equal temperature obtained from cooling: in figure 6 one can see that all features are less sharp in the latter case, with some peaks even missing with respect to the perfect crystal case. As can be seen in the corresponding panels in figure [2] an evident "hysteresis" characterizes the heating cycles with respect to the cooling sequences.

\section{Quenching and glass transition}

Quenching of the liquid at different pressures is carried out for $N=1000$ and 864, starting from an initial temperature $T=1950 \mathrm{~K}$, either through a sequence of $\Delta T=150 \mathrm{~K}$ decrease steps, or through a sudden $\Delta T=1000 \mathrm{~K}$ drop. Temperature variations are imposed over 20000 simulation steps, followed by 10000 steps of free evolution. The quenching paths are displayed in figure 1 (top and middle panels) while the volume and enthalpy behavior is visible in figure 2 (top and middle panels).

The onset of a glassy phase during both quenching procedures is visually documented in the snapshots shown in figure [5] and is proved by several evidences on thermodynamic, structural and dynamical quantities. We first observe that the $V$ and $H$ patterns in figure 2 show a glass branch running above the crystallization line; moreover, at variance with what we have observed during the slow cooling, the radial distibution functions in figure 3] do not develop the peak structure of the fcc arrangement at low temperatures; rather, down to $\simeq 300 \mathrm{~K}$ they exhibit the twofold structure of the second peak typical of the glass. Such a structural evidence has a counterpart in the diffusion coefficient behavior which, as visible in figure 4 does not exhibit the drop associated to the onset of crystallization process, but manifests only a change of slope. On the other hand, the mean square displacements show a plateau for temperatures $T \leq 1100 \mathrm{~K}$, signalling structural arrest, also confirmed by the shape variations of the velocity autocorrelation function with the temperature (see figure 7). Changes of slope as the temperature decrease, typically associated to the glass transition, occur also in $V$ and $H$ (figure 2), as well as 
in the thermal expansivity $\alpha$, the specific heat $C_{\mathrm{p}}$, and the Wendt-Abraham ratio $R$ of the first peak to the first minimum height in $g(r)$ (figure \&).

Evidence of the glass formation also comes from the shear viscosity which we have calculated via the GreenKubo relation 41]:

$$
\eta_{\mathrm{sh}}=\frac{1}{V k_{\mathrm{B}} T} \int_{0}^{\infty}\left\langle\sigma^{x y}(0) \sigma^{x y}(t)\right\rangle \mathrm{d} t
$$

where

$$
\sigma^{x y}=\sum_{i=1}^{N}\left[m_{i} v_{i}^{x} v_{i}^{y}+\frac{1}{2} \sum_{j \neq i} x_{i j} f_{y}\left(r_{i j}\right)\right]
$$

is the off-diagonal component of the stress tensor, $v_{i}^{x}$ and $x_{i j}$ represent, respectively, the $x$ component of the velocity and separation distance, $r_{i j}$, between molecules' center-of-mass $i$ and $j$, and $f_{y}$ is the $y$ component of the force exerted on atom $i$ by atom $j$. As can be appreciated in figure $9 \eta_{\text {sh }}$ exhibits a marked increase across the glass transition region and displays an Arrhenius behavior as a function of $T$ (see inset), indicating that the glass formed is a "strong" one 42]; the orientational glass of $\mathrm{C}_{60}$ is similarly "strong" 29, 30].

The glass transition parameters are obtained from the intersection of the two (extrapolated) branches in the $V, H, C_{\mathrm{P}}, \alpha$ and $R$ patterns as functions of the temperature (see reference [2] for details): we estimate at $P=3.5 \mathrm{MPa} T_{\mathrm{g}} \simeq 1100 \mathrm{~K}$ and $\rho_{\mathrm{g}} \simeq 1.168 \mathrm{~nm}^{-3}$.

As visible from figure 2 a glass transition is observed also at pressures higher than $3.5 \mathrm{MPa}$, at the cost to increase the quenching rate with respect to $1.5 \times 10^{12} \mathrm{~K} / \mathrm{s}$ adopted at $3.5 \mathrm{MPa}$. For instance, at $P=250 \mathrm{MPa}$ no glassy phase is obtained if $\Delta T \leq 300 \mathrm{~K}$ over 20000 time steps. Glass transition temperatures and densities at different pressures are reported in table प It is immediate to verify that, similarly to what observed with the crystallization transitions, the glass transitions (at different pressures) occur for states falling at the crossing of the quenching paths with the $\rho(T)$ locus defined by equation (5), where $\eta_{\mathrm{g}}=\eta_{\mathrm{g}}^{\mathrm{HS}}$. We shall comment on these evidences in the next section.

The quenching rates we adopt in our simulations are much higher than those presently achieved in experiments (typically $10^{8} \mathrm{~K} / \mathrm{s}$ ). We have however preliminary simulative evidences [43] that in equimolar Girifalco $\mathrm{C}_{60} / \mathrm{C}_{70}$ and $\mathrm{C}_{60} / \mathrm{C}_{96}$ mixtures crystallization does not occur even upon cooling rate one or two order of magnitude lower than those adopted here; the $\mathrm{C}_{60} / \mathrm{C}_{70}$ mixture, for instance, remains liquid during the cooling procedure down to $1100 \mathrm{~K}$, a temperature lower than that at which amorphization of pure $\mathrm{C}_{60}$ fullerite occurs 22 ] (see also note [26]). These findings make it plausible that a glassy phase might be formed experimentally at least from mixed fullerene systems.

\section{DISCUSSION AND CONCLUSIONS}

The effective packing at crystallization for different pressures have values close to the packing fraction of hard

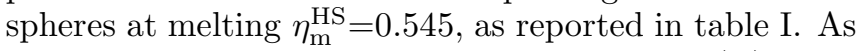
a matter of fact, it thus apperas that the $\eta_{\mathrm{m}}(T)$ locus introduced in equation (4) acts as an interpolation curve among the transition points determined a posteriori, and can be used to forecast crystallization temperatures and densities at pressures other than those here considered. A similar evidence is found for the effective packing at the glass transition $\eta_{\mathrm{g}}(T)$ of equation (5) also reported in the table, that is quite close to the hard sphere glass transition value, $\eta_{\mathrm{g}}^{\mathrm{HS}}=0.58[37$. As for the onset of crystallization and glass transitions, an obvious deduction is that the Girifalco model follows rather closely a hardsphere-like behavior. Our evidences, however, allow us to get a deeper insight into the role of the $\rho_{\mathrm{m}}(T)$ and $\rho_{\mathrm{g}}(T)$ loci. Indeed, we have found that when supercooling is pushed beyond $\rho_{\mathrm{m}}(T)$ the system invariably crystallizes; by contrast, a state close to, but on the left of $\rho_{\mathrm{m}}(T)$, remains in the supercooled phase even over the longest simulation run we could perform, namely 12 million simulation steps, corresponding to $60 \mathrm{~ns}$. It thus appears that the $\rho_{\mathrm{m}}(T)$ locus approximately corresponds to an instability boundary of the metastable region. This observation also emerges from Monte Carlo calculations of the free energy of the Girifalco model at $T=2100 \mathrm{~K}[13$ ] (see inset of figure 1); in fact, $\rho_{\mathrm{m}}(2100 \mathrm{~K})$ coincides with the point where the free energy shows an inflection point associated with a sudden drop of the pressure of the simulation sample, i.e. with a mechanical instability of the system. A similar property might characterize the free energy branch generated by approaching the metastable region from the solid side and since the interval separating the two branches in figure 1 is quite narrow, it is conceivable that our simulation strategy is unable to discriminate between the densities at the crystallization and melting instabilities. It is interesting to observe that in Molecular Dynamics simulations of the Lennard-Jones potential, crystallization occurs much beyond the melting line, deeply inside the solid region [44]. Indeed we have carried out MD simulations of the same potential and verified that states located inside the metastable fluidsolid region, do not undergo crystallization even after 35 million time steps $(\simeq 180 \mathrm{~ns})$.

Our results fot the glass transition positively agree with the predictions made by Foffi and coworkers [7] on the glass transition in protein solutions modeled through the attractive hard-core Yukawa (HCY) potential. These authors find that for inverse decay length $z$ of the Yukawian term spanning from 5 to 60 times the reciprocal of the particle diameter, the glass transition line is an almost vertical locus with a $T=\infty$ asymptote $\eta=\eta_{\mathrm{g}}^{\mathrm{HS}}=0.58$. On the other hand, as discussed in [45], the physical properties of the Girifalco model can be reasonably reproduced by a Yukawa potential with $z \simeq 4$ (though with moderate differences in the phase diagram), close indeed 
to the lowest value investigated in reference [7]. A qualitative agreement thus emerges between the prediction of the glass transition in the two different systems. In the HCY model the formation of "repulsive" and "attractive" glasses depends on the value of $z$ 7]; since at $z$ as low as five only the repulsive glass would be formed, we identify our $\mathrm{C}_{60}$ "positional" glass as a repulsive one.

In conclusion, we have documented that effective hardcore exclusion effects play an important role for the determination of the glass transition in a short-range potential well suited to model various fullerenes. Mode Coupling Theory calculations for the onset of the glass transition in a short-range model of globular protein solutions lead to similar conclusions at least for relatively low values of the decay potential parameters. In this context, it could be worth to carry out specific Mode Coupling calculations for the present model fullerene in order to compare the structural arrest line thereby predicted with our re- sults for the glass line based on simulations and $\rho_{\mathrm{g}}(T)$ locus predictions. Moreover, colloidal suspensions and colloid-polymer mixtures show a behavior characterized by several similarities with the observations reported in this work [6]. We argue that the basic equation (5) might hold to a high accuracy also for other interaction potentials, and hence it could provide a simple framework for a qualitative but immediate prediction of the glass line in a variety of model systems.

\section{Acknowledgments}

This work has been done in the framework of the Marie Curie Network on Dynamical Arrest of Soft Matter and Colloids, Contract Nr MRTN-CT-2003-504712.
[1] Rosenbaum, D. F.; Zamora, P. C.; Zukoski, C. F. Phys. Rev. Lett. 1996, 76, 150.

[2] Lomakin, A.; Asherie, N.; Benedek, G. B. J. Chem. Phys. 1996, 104, 1646.

[3] Piazza, R.; Peyre, V.; Degiorgio, V. Phys. Rev. E 1998, 58, R2733.

[4] Poon, W. C. K. Phys. Rev. E 1997, 55, 3762.

[5] ten Wolde, P. R.; Frenkel, D. Science 1997, 277, 1975.

[6] Louis, A. A. Phil. Trans. R. Soc. Lond. A 2001, 359, 939; Pham, K. N.; Egelhaaf, S. U.; Pusey, P. N.; Poon, W. C. K. Phys. Rev. E 2004, 69, 011503.

[7] Foffi, G.; McGullagh, G. D.; Lawlor, A.; Zaccarelli, E.; Dawson, K. A.; Sciortino, F.; Tartaglia, P.; Pini, D.; Stell, G. Phys. Rev. E 2002, 65, 031407; Dawson, K. A. Current Opinion in Colloid and Interface Science 2002, 7, 218.

[8] Pellicane, G.; Costa, D.; Caccamo, C. J. Phys. Chem. B 2004, 104, 7538 .

[9] Cheng, A.; Klein, M. L.; Caccamo, C. Phys. Rev. Lett. 1993, 71, 1200.

[10] Hagen, M. H. J.; Meijer, E. J.; Mooij, G. C. A. M.; Frenkel, D.; Lekkerkerker H. N. W. Nature 1993, 365, 425.

[11] Ashcroft, N. W. Nature 1993, 365, 387.

[12] Hasegawa M.; Ohno, K. J. Phys.: Cond. Matter 1997, 9, 3361; J. Chem. Phys. 1999, 111, 5955.

[13] Abramo, M. C.; Caccamo, C.; Costa, D.; Pellicane, G. Europhys. Lett. 2001, 54, 468.

[14] Costa, D.; Pellicane, G.; Abramo, M.C.; Caccamo, C. J. Chem. Phys. 2003, 118, 304.

[15] Chayen, N. E. Trends in Biotechnology 2002, 20, 98.

[16] McPherson, A. Preparation and Analysis of Protein Crystals; Krieger: Malabar, 1982.

[17] Broide, M.; Tominc, T. M.; Saxowsky, M. D. Phys. Rev. E 1996, 53, 6325. Grigsby, J. J.; Blanch, H. W.; Prausnitz, J. M. Biophys. Chem. 2001, 91, 231.

[18] Eckert T.; Bartsch E. Phys. Rev. Lett. 2002, 89, 125701.

[19] Chen, S. H.; Chen, W. R.; Mallamace, F. Science 2003, $300,619$.

[20] Pham K. N.; Egelhaaf S. U.; Pusey P. N.; Poon W. C. K.
Phys. Rev. E, 2004, 69, 011503.

[21] Girifalco, L. F. J. Phys. Chem. 1992, 96, 858.

[22] Stetzer, M. R.; Heiney, P. A.; Fischer, J. E.; McGhie, A. R. Phys. Rev. B 1997, 55, 127.

[23] Xu, C.; Scuseria, G. E. Phys. Rev. Lett. 1994, 72, 669; Kim, S. C.; Tomanek, D. Phys. Rev. Lett. 1994, 72, 2418.

[24] Sundar, C. S.; Bharathi, A.; Hariharan, Y.; Janaki, J.; Sankara Sastri, V.; Radhakrishnan, T. Solid State Comm. 1992, 84, 823.

[25] Abramo, M. C.; Caccamo, C. J. Chem. Phys. 1997, 106, 6475; Ruberto, R.; Abramo, M. C. J. Chem. Phys. 2004, submitted for publication.

[26] Fullerite (crystalline $\mathrm{C}_{60}$ ) heated at $T>1200 \mathrm{~K}$, mostly transforms in amorphous carbon due to the disruption of the $\mathrm{C}_{60}$ cages (see ref [22]). The latter, however, are predicted to be stable up to 3500-4000 K 23]. Solid state effects through molecule collision [24], but also and alternatively residual impurities trapped in the crystalline matrix [25], might trigger the cage instability.

[27] Alemany, M. M. G.; Rey, C.; Dieguez, O.; Gallego, L. J. J. Chem. Phys. 2000, 112, 10711.

[28] Abramo, M. C.; Caccamo, C. Costa, D; Ruberto, R, J. Phys. Chem. B 2004, 108, 13576.

[29] Gugenberger, F.; Heid, R.; Meingast, C.; Adelmann, P.; Braun M.; Wuhl, M.; Haluska, M.; Kuzmany, H. Phys. Rev. Lett. 1992, 69, 1774.

[30] Matsuo, T.; Tsuo, T.; Suga, H.; David, W. I. F.; Ibberson, R. M.; Benrier, P.; Zahab, A.; Fabre, C.; Rassat, A.; Dworkin, A. Solid State Comm. 1992, 83, 711.

[31] Andersen, H. C. J. Chem. Phys.1980, 72, 2384.

[32] Weeks, J. D.; Chandler, D.; Andersen, H. C. J. Chem. Phys. 1971, 54, 5237.

[33] Barker, J. A.; Henderson, D. J. Chem. Phys. 1967, 47, 2856.

[34] Caccamo, C. Phys. Rep. 1996, 274, 1.

[35] Alder, B. J.; Wainwright, T. E. J. Chem. Phys. 1959 31, 459; Hoover, W. G.; Ree, F. H. J. Chem. Phys., 1968, 49, 3609.

[36] Gruhn, T.; Monson, P. A. Phys. Rev. E 2001, 64, 061703 and references therein. 
[37] Woodcock, L. V. Ann. N. Y. Acad. Sci. 1981, 37, 274.

[38] Shumway, S. L.; Clarke, A. S.; Jonsson, H. J. Chem. Phys. 1995, 102, 1796.

[39] Caccamo, C.; Costa, D.; Fucile A. J. Chem. Phys. 1997, 106, 255.

[40] Costa, D.; Ballone, P.; Caccamo, C. J. Chem. Phys.2002, 116, 3327.

[41] Hansen J-P and McDonald I R Theory of Simple Liquids 2nd edition; Academic Press; London 1986.

[42] Angell, C. A. J. Non-Cryst.Solids 1991, 131-133, 13.

[43] Abramo, M. C.; Caccamo, C.; Ruberto, R. (unpublished).

[44] Nosè, S.; Yonezawa, F. Solid State Comm. 1985, 56, 1005; J. Chem. Phys. 1986, 840, 1803.

[45] Hagen, M. H. J.; Frenkel, D. J. Chem. Phys. 1994, 101, 4093. 

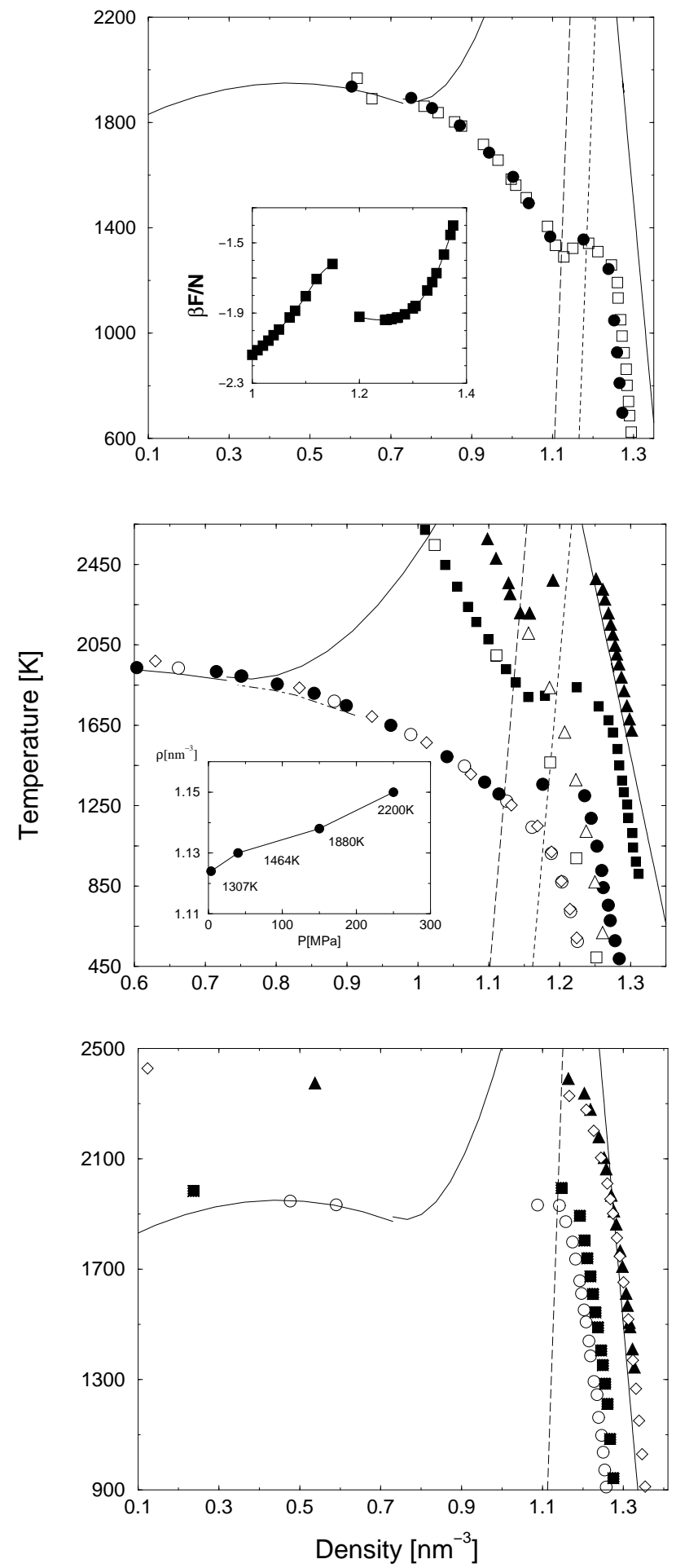

FIG. 1: Top: Continuous lines: phase diagram of $\mathrm{C}_{60}$ [14] with superimposed the $\rho_{\mathrm{m}}(T)$ [long-dashed line, equation (4)] and $\rho_{\mathrm{g}}(T)$ [short-dashed line, equation (5)] loci introduced in this work. Circles and squares: cooling with 1000 and 864 particles at $P=3.5 \mathrm{MPa}$, respectively. Inset: Helmholtz free energy vs $\rho$ at $T=2100 \mathrm{~K}$ [14]. Middle: Effect of increasing pressure on the cooling and quenching paths. Full circles, squares and triangles: cooling paths with $N=1000$ at pressure $P=3.5,150$ and $250 \mathrm{MPa}$, respectively; open circles, squares and triangles: quenching with $N=1000$ at $P=3.5,150$ and $250 \mathrm{MPa}$; diamonds: quenching at $3.5 \mathrm{MPa}$ with 864 particles. The dot-dashed line indicates the metastable portion of the binodal [39]. Inset: crystallization density as a function of the pressure and corresponding temperatures. Bottom: Heating paths of the defective and perfect crystals. Circles and squares: heating of the defective crystal with $N=1000$ and 864, respectively; diamonds and triangles: heating of the perfect crystal with $N=864$ at $P=3.5 \mathrm{MPa}$ and $12 \mathrm{MPa}$, respectively. 

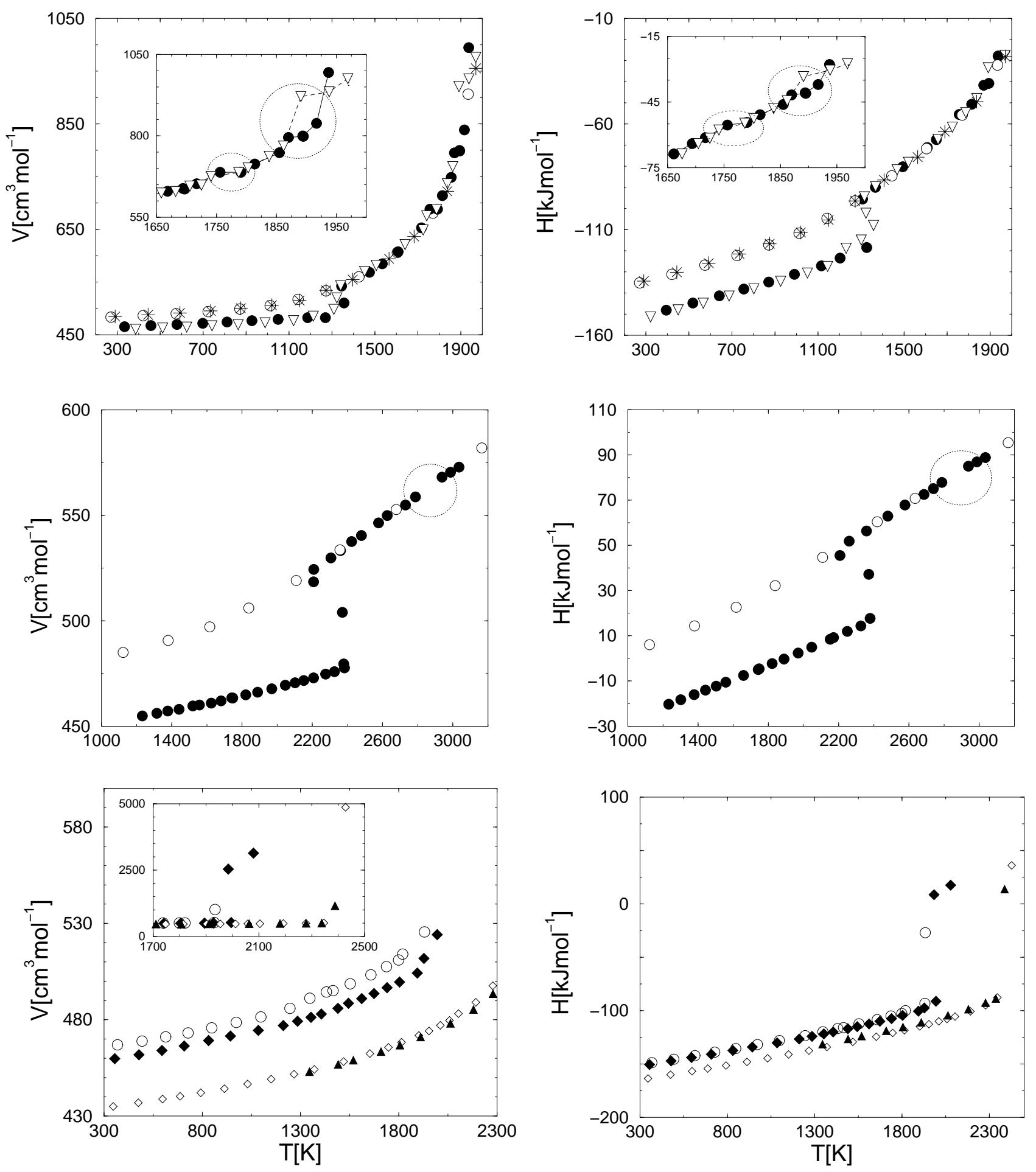

FIG. 2: Top: Volume (left) and enthalpy (right) as functions of the temperature in cooling and quenching cycles at $P=3.5 \mathrm{MPa}$. Full circles, open triangles: cooling with $N=1000$ and 864, respectively; open circles, asterisks: quenching with $N=1000$ and 864, respectively. Inset: expansion of the crossing zones (encircled) of the freezing and binodal line during cooling. Middle: $V$ (left) and $H$ (right) vs $T$ in cooling (full circles) and quenching (open circles) cycles with $N=1000$ at $P=250$ MPA. Bottom: $V$ (left) and $H$ (right) vs $T$ in heating cycles. Open circles, full diamonds: heating of the defective crystal with $N=1000$ and 864 at $P=3.5 \mathrm{MPa}$, respectively; open diamonds, full triangles: heating of the perfect crystal with $N=864$ particles at $P=3.5$ and $12 \mathrm{MPa}$,respectively. Inset: expansion of the volume scale around the transition to the liquid (for $N=1000$ at $3.5 \mathrm{MPa}$, open circles), and the sublimation to a highly expanded vapor phase (for all other cases). 

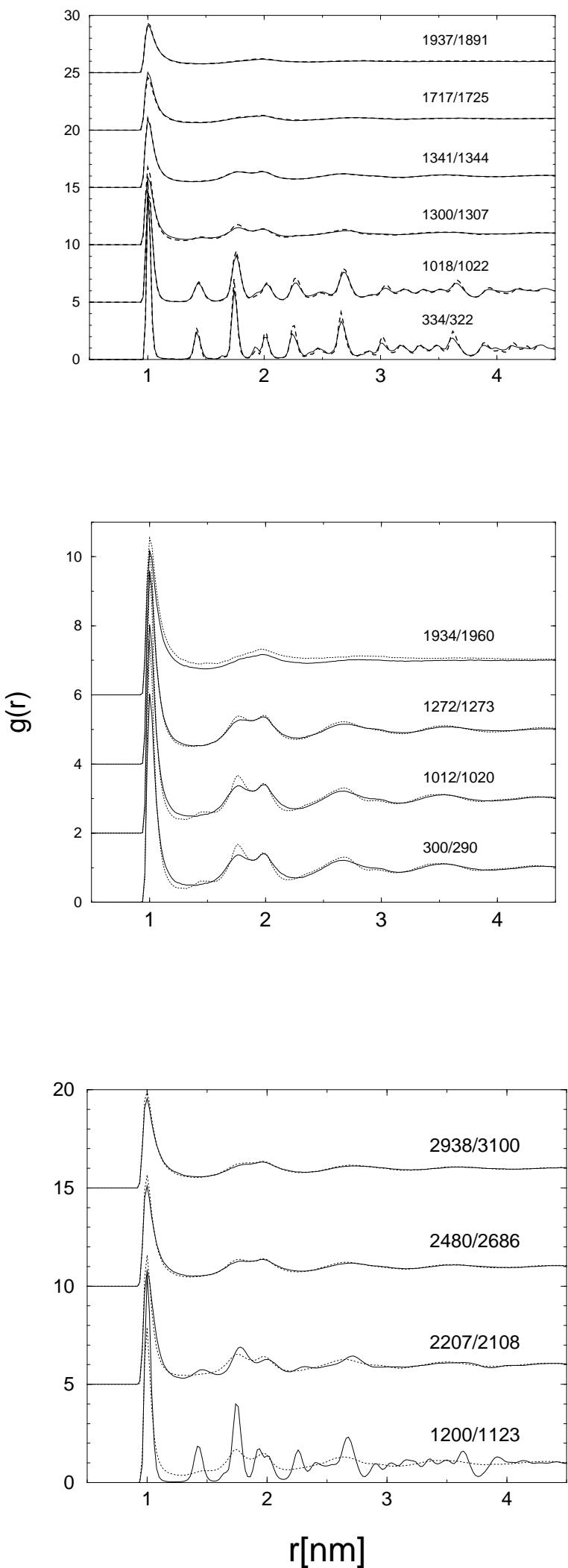

FIG. 3: Top: evolution of $g(r)$ with the temperature during cooling at $3.5 \mathrm{MPa}$, with $N=1000$ (full line) and $N=864$ (dashed line). Temperatures aside the curves refer to the $N=1000$ (left) and 864 (right). Middle: same as in the top panel for quenching cycles. Bottom: Cooling (full line) and quenching (dashed line) for $N=1000$ at $P=250 \mathrm{MPa}$. 

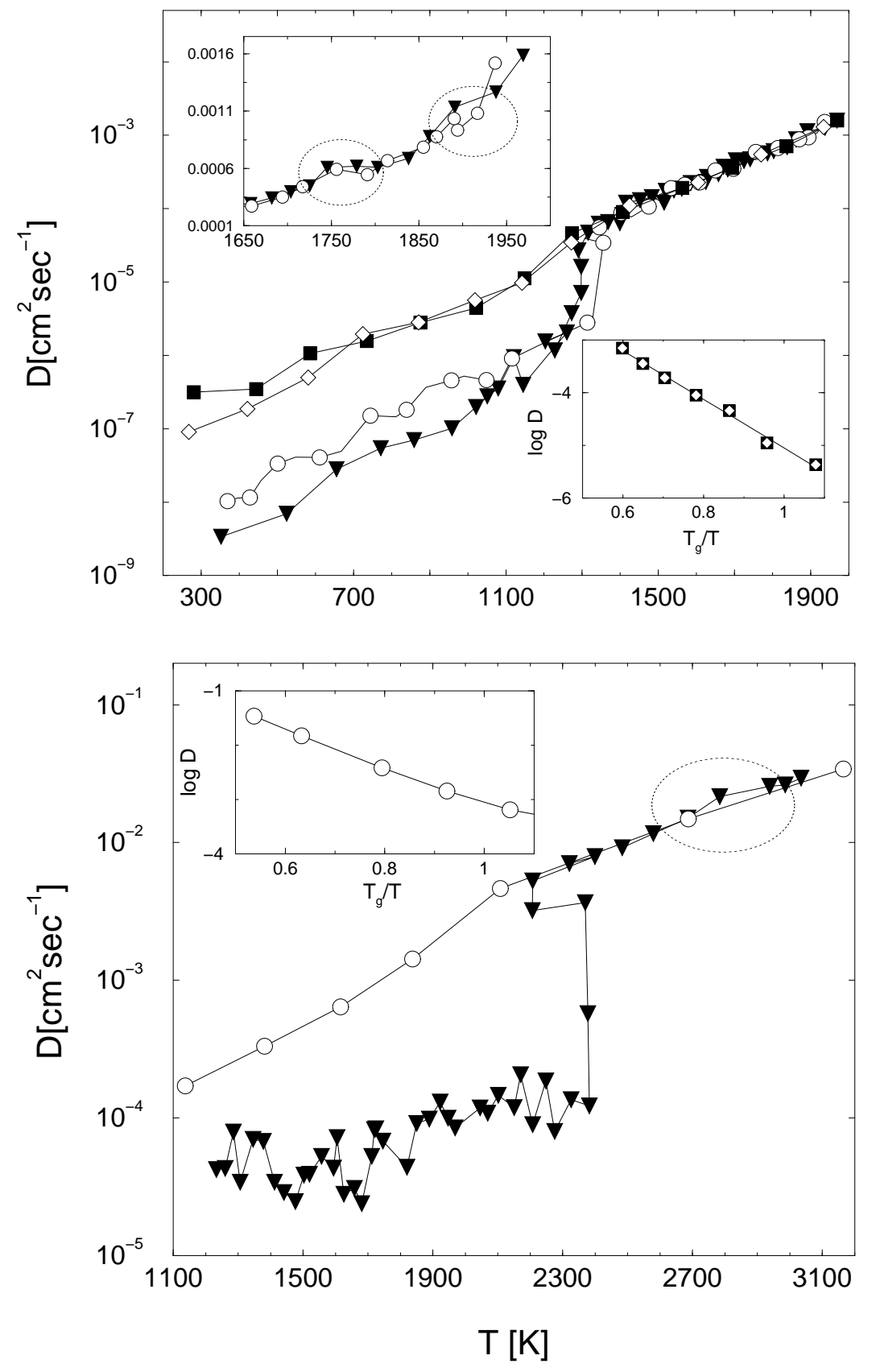

FIG. 4: Top: Diffusion coefficient of cooling (open circles, full triangles) and quenching (open diamonds and full squares) cycles for $N=1000$ and 864 , at $3.5 \mathrm{MPa}$, respectively. Upper inset: linear scale expansion of the $D$ behaviour at the crossing of the freezing and binodal lines; lower inset: Arrhenius plot of the diffusion coefficient in quenching cycles. Bottom: Diffusion coefficient in cooling (full triangles) and quenching (open circles) cycles at $P=250 \mathrm{MPa}$ for $N=1000$. Inset: Arrhenius plot of $D$ in the quenching cycles. 

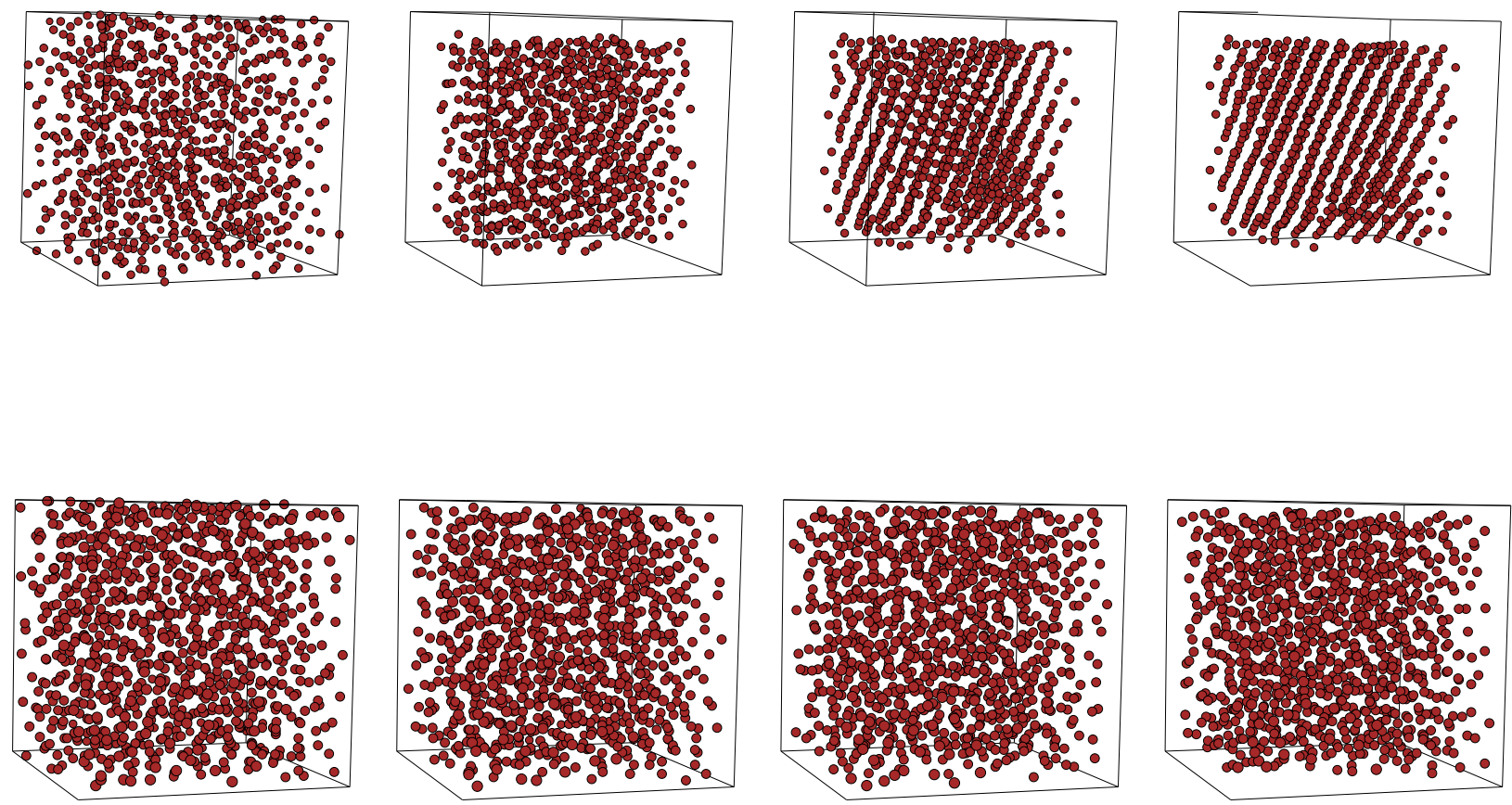

FIG. 5: Snapshots of the $N=1000$ sample during the cooling (top) and the quenching (bottom) sequence at $P=3.5 \mathrm{MPa}$ and $T=1937,1307,1152$, and $300 \mathrm{~K}$ (from left to right).

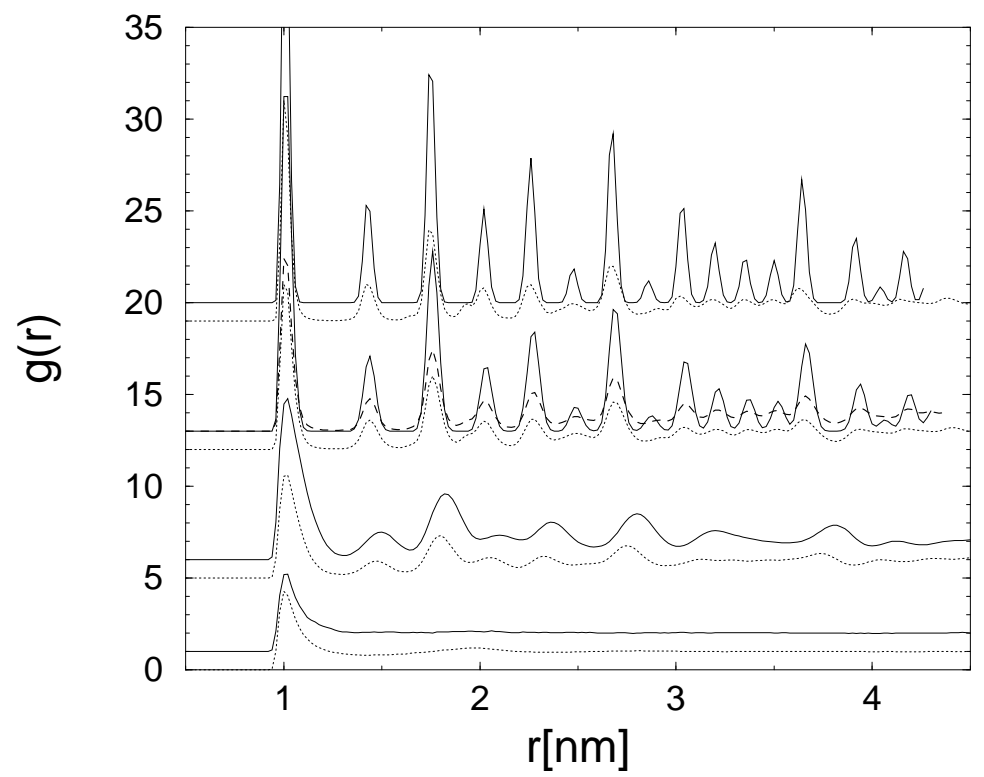

FIG. 6: Evolution of $g(r)$ during the heating sequence for the perfect crystal with $N=864$ (full line) at $T=598,1030$, 2346 , and $2391 \mathrm{~K}$ (from top to bottom), and for the defective crystal with $N=1000$ (dotted line) at $T=584,1037,1931$, and $1934 \mathrm{~K}$. The dashed line is $g(r)$ at $T=1022 \mathrm{~K}$, as obtained by cooling the $N=864$ sample from high temperature (1937 K). The comparison with the $1037 \mathrm{~K}$ heating case enlights the smoother features resulting from cooling. 

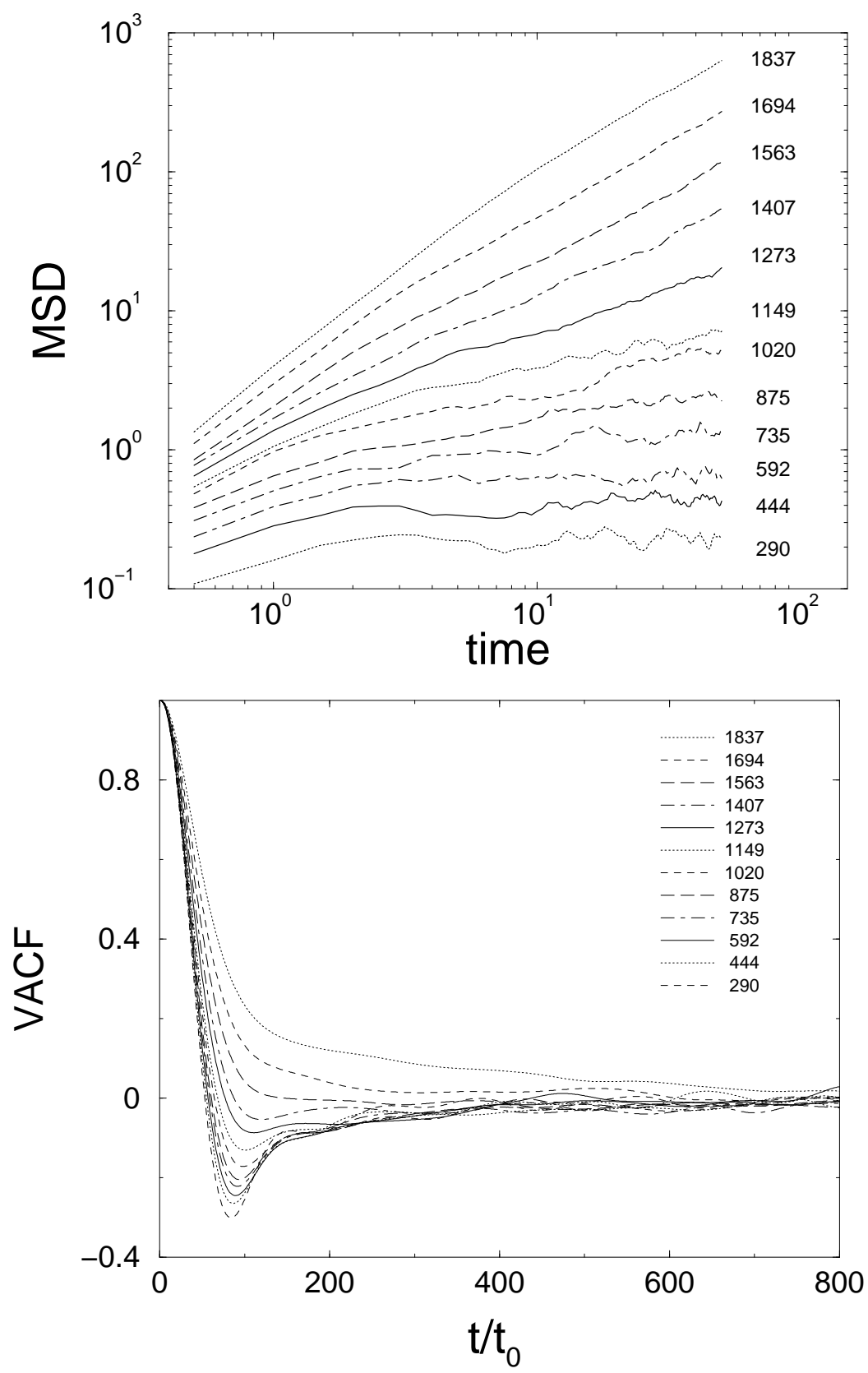

FIG. 7: Top: Mean square displacement (in $\AA / \mathrm{s}$ units) in the $N=864$ sample as a function of the simulation time (in picoseconds) during the quenching sequence at $P=3.5 \mathrm{MPa}$. Bottom: Evolution of the velocity autocorrelation function during the same sequence $\left(t_{0}=\right.$ time step). 

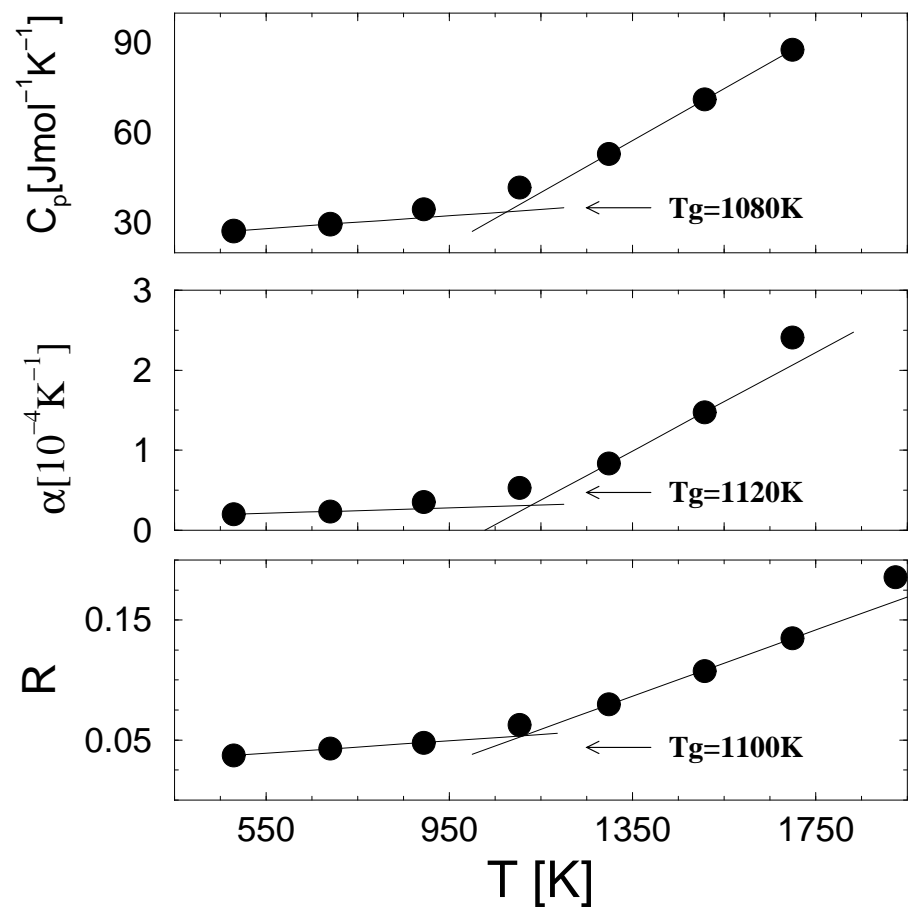

FIG. 8: Heat capacity (top), thermal expansion (middle), and Wendht-Abraham ratio (bottom) as functions of the temperature during the quenching sequence of the $N=864$ sample at $P=3.5 \mathrm{MPa}$.

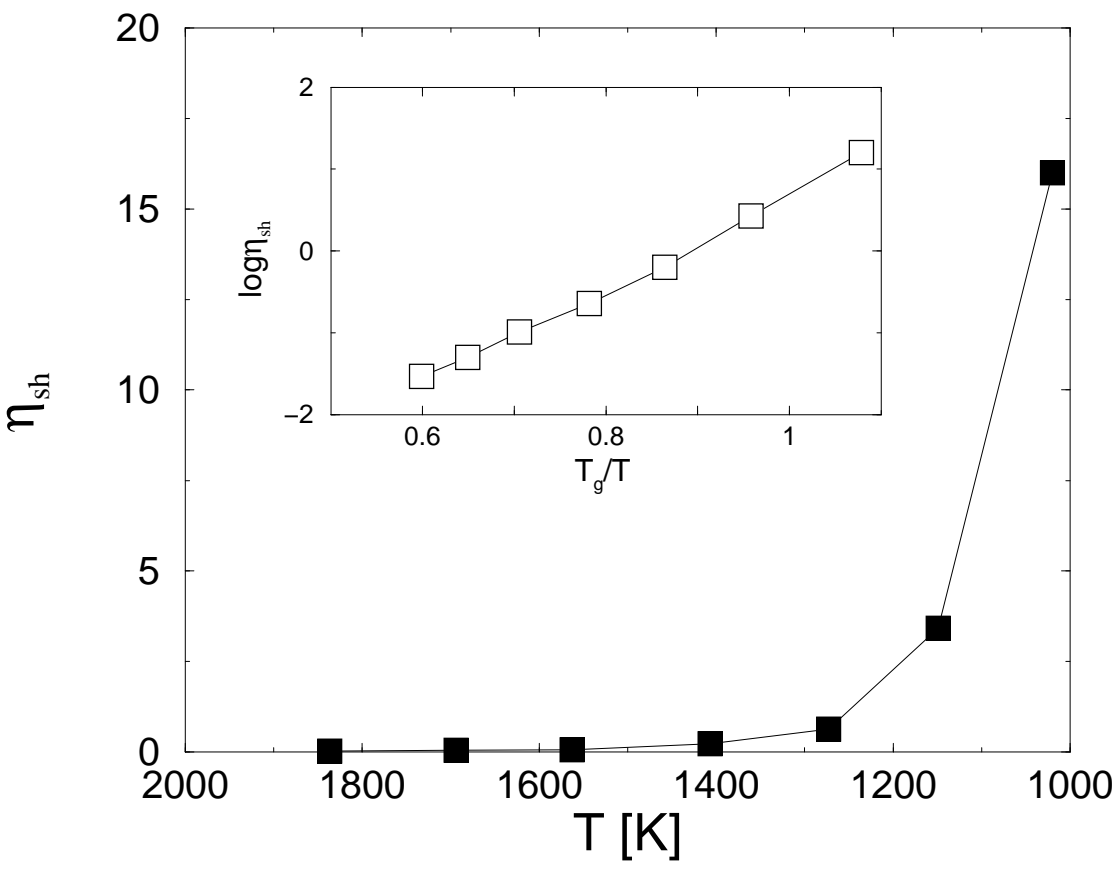

FIG. 9: Shear viscosity vs temperature during a quenching sequence of the $N=864$ sample at $P=3.5 \mathrm{MPa}$. The inset shows the Arrhenius behaviour in the approach to the glass temperature. 